J. Lake Sci. (湖泊科学), 2021, 33(6): 1898-1905

DOI 10. 18307/2021. 0623

(C) 2021 by Journal of Lake Sciences

\title{
三峡水库运用后荆江段非均匀悬沙恢复特性
}

\author{
冯 雪, 夏军强 ${ }^{* *}$, 周美蓉, 邓珊珊 \\ (武汉大学水资源与水电工程国家重点实验室,武汉 430072)
}

\begin{abstract}
摘 要: 三峡工程运用后, 坝下游荆江段来沙量大幅度减小, 处于严重的不平衡输沙状态, 次饱和水流冲刷河床使悬沙量 沿程恢复. 基于实测水沙资料, 分析了三峡工程运用后荆江段非均匀悬沙恢复特点. 提出了恢复效率的概念用以表征悬 移质沿程恢复的程度, 并根据实测水沙资料计算了荆江段 1994-2017 年非均匀悬沙的恢复效率. 结果表明: 三峡工程运 用前, 荆江段各粒径组悬沙恢复效率绝对值均接近 0 , 故该时期内各粒径组泥沙冲淤幅度不大; 三峡工程运用后, 各粒径 组悬沙恢复效率绝对值均明显增大, 且粗沙 $(d>0.125 \mathrm{~mm})$ 恢复效率绝对值远大于细沙 $(d<0.125 \mathrm{~mm})$, 故粗沙恢复程度 更高. 这主要是由于荆江段床沙组成中粗沙部分含量大, 而细沙含量小. 最后建立了三峡水库蓄水后非均匀悬沙恢复效 率与来水来沙条件 (来沙系数) 的关系, 结果表明: 各粒径组悬移质恢复效率均与来沙系数呈正相关关系, 全沙、细沙和中 沙的决定系数 $\left(R^{2}\right)$ 分别为 $0.89 、 0.67$ 和 0.69 , 相关性较高, 故荆江段各粒径组悬移质泥沙恢复效率较大程度上受到来水 来沙条件的影响.
\end{abstract}

关键词: 非均匀悬沙; 不平衡输移;恢复效率;荆江河段; 三峡工程

\section{Transport characteristics of non-uniform suspended sediment in the Jingjiang Reach after the Three Gorges Project operation*}

Feng Xue, Xia Junqiang ** ${ }^{*}$ Zhou Meirong \& Deng Shanshan

( State Key Laboratory of Water Resources and Hydropower Engineering Science, Wuhan University, Wuhan 430072, P.R.China)

\begin{abstract}
The amount of suspended sediment entering the Jingjiang Reach of the middle Yangtze River has been greatly reduced owing to the operation of the Three Gorges Project (TGP), which caused the sediment transport of the reach at a severe non-equilibrium state, and the suspended sediment concentration recovered during the channel scour process. Therefore, the characteristics of non-equilibrium transport of non-uniform suspended sediment were analyzed based on the measured flow and sediment data. In addition, the recovery efficiency was proposed to represent the recovery speed for suspended sediment load, and the corresponding values were calculated annually from 1994 to 2017 based on the measured data of the Jingjiang Reach. Calculated results show that the suspended sediment recovery efficiency of all particle-size groups was very close to zero before the TGP operation, which means that the channel deformation was small during this period. However, the recovery efficiency of each particle-size group increased greatly after the operation of TGP, and the recovery efficiency of coarse sediment $(d>0.125 \mathrm{~mm})$ was much higher than that of fine sediment $(d<0.125 \mathrm{~mm})$. This is mainly because the content of coarse sediment in bed-material of Jingjiang Reach is large, while the content of fine sediment is relatively small. Finally, empirical relationship was developed between the recovery efficiency and incoming flow-sediment regime, which shows that the recovery efficiency of each group was negatively related to incoming sediment coefficient, with the correlation coefficient for the total, fine and coarse sediment being $0.89,0.67$ and 0.69 , respectively. Therefore, it can be concluded that the recovery efficiency of suspended sediment was significantly affected by the incoming flow and sediment conditions.
\end{abstract}

Keywords: Non-uniform suspended sediment; non-equilibrium transport; recovery efficiency; Jingjiang Reach; Three Gorges Project

\footnotetext{
* 2020-10-31 收稿;2021-04-25 收修改稿.

国家自然科学基金项目 (51725902，U2040215，52009095)联合资助.

** 通信作者; E-mail:xiajq@whu.edu.cn.
} 
冲积河流中上游来沙量往往不会恰好等于当地的水流挟沙力,不平衡输沙现象十分普遍,河床往往会 通过冲淤调整同时自动调整水流挟沙力的大小, 使河床逐渐趋于输沙平衡状态 ${ }^{[1-2]}$. 水库运用后, 泥沙淤积 在库区,下泄水流的含沙量大幅度下降,坝下游河段往往会发生长距离不平衡输沙过程 ${ }^{[2-4]}$. 而目前该过程 往往难以精准预测, 主要是因为对不平衡输沙规律尤其是非均匀沙的认识不够, 故研究坝下游非均匀沙不 平衡输移规律, 具有重要的理论意义及实际应用价值.

近几十年来,国内外学者对坝下游河道不平衡输沙过程进行了较多的研究. 早期部分学者主要通过求 解悬移质紊动扩散方程来模拟不平衡输沙过程, 但通过该方法求得的含沙量恢复饱和距离很短, 与实际冲 刷距离存在较大差异 ${ }^{[5]}$. 为了预报实际问题, 部分学者从垂线平均含沙量沿程变化出发, 引人恢复饱和系 数, 并建立不平衡输沙方程 ${ }^{[6]}$, 对不平衡输沙过程进行计算. 但由于缺乏对恢复饱和系数 $\alpha$ 的物理本质及变 化规律的认识, 该方法也具有一定的局限性. 目前关于坝下游悬移质输移特性及水流含沙量恢复特性的认 识,多源于对已建大坝下游冲刷发展的观测与分析,主要包含两类观点.

一类观点涉及粗细泥沙交换理论. 部分学者认为坝下游长距离冲刷主要是由于水流挟沙力沿程增加. 如钱宁等 ${ }^{[7]}$ 认为床沙粒径沿程变细导致水流挟沙力沿程增加, 是坝下游长距离冲刷的根本原因. 韩其为 ${ }^{[8]}$ 认为含沙量恢复距离很长主要是因为河床组成沿程变细, 悬移质泥沙与床沙颗粒之间的交换使得悬沙级配 沿程变细, 挟沙力沿程增加, 从而导致细颗粒泥沙恢复距离较长. 也有部分学者如刘金梅等 ${ }^{[9]}$ 从悬移质扩散 方程出发,提出表层床沙粗化的概化计算模式及床沙交换速率计算公式,并采用黄河下游资料对该公式进 行验证, 发现表层床沙冲刷粗化致使挟沙力降低, 是导致不平衡输沙距离大大延长的重要因素. 而另一类观 点主要涉及床沙组成问题. 如 $\mathrm{Li}$ 等 ${ }^{[10]}$ 基于对已建大坝下游的实测资料分析后认为, 细颗粒泥沙恢复距离较 长主要是因为床沙补给不足. 陈飞等 ${ }^{[11]}$ 认为水库运用后近坝段各组泥沙均发生剧烈冲刷, 河床粗化显著; 距离大坝较远的河段, 只要上游河段有充足的泥沙补给, 各粒径组泥沙均能基本达到饱和; 并指出水库下游 发生长距离冲刷主要是因为床沙补给不足, 尤其是细沙. 据此, 有许多学者对坝下游河段非均匀沙的不平衡 输移特性进行了研究. 例如, 郭小虎等 ${ }^{[12]}$ 根据实测资料分析指出, 三峡大坝下游粗沙 $(d>0.125 \mathrm{~mm})$ 在荆江 河段基本可达饱和, 其主要是因为荆江段河床中存在大量较粗泥沙, 而细沙含量很小, 导致河床补给不足. 杨云平等 ${ }^{[13]}$ 利用 1987-2014 年的实测水沙数据, 分析了三峡大坝下游非均匀沙的输移过程, 发现蓄水后坝 下游 $d<0.125 \mathrm{~mm}$ 粒径组含沙量沿程递增, 且小于蓄水前; $d>0.125 \mathrm{~mm}$ (粗颗粒) 含沙量在宜昌一监利河段得 到补给, 其下游为淤积趋势. 陈建国等 ${ }^{[14]}$ 分析了黄河下游实测水沙资料, 探讨了三门峡水库不同典型运用 时段非均匀沙的冲淤特点, 发现当水库下泄清水, 河床冲刷时, 水流含沙量逐渐恢复, 细沙恢复较慢, 恢复距 离长, 而中、粗泥沙恢复距离短. 虽然迄今有很多学者对坝下游河段非均匀沙不平衡输移过程进行了大量研 究, 但鲜有研究涉及定量分析不同粒径组悬沙的恢复程度及其与来水来沙条件的关系. 故有必要进一步研 究坝下游典型河段非均匀沙含沙量的沿程恢复特性, 从而提高对坝下游河段不平衡输沙规律的认识, 为河 床冲淤过程预测提供理论依据.

因此本文在已有研究的基础上,根据 1994-2017 年荆江段的实测水沙资料,分析三峡水库运用前后该 河段非均匀悬移质泥沙的不平衡输移特点; 提出恢复效率的概念用于表征悬移质含沙量恢复的程度,计算 蓄水前后荆江段分组沙的恢复效率,并建立其与河段进口来水来沙条件的相关关系.

\section{1 非均匀悬沙不平衡输移特点}

三峡水库运用后, 泥沙淤积在库区, 坝下游荆江段悬移质含沙量大幅下降, “清水” 冲刷河床使得含沙量 逐渐恢复从而趋向平衡输沙状态. 天然河道中的泥沙往往为非均匀沙, 各粒径组含沙量也不尽相同, 因此不 同粒径组悬沙的沿程恢复特点存在较大差异, 仅研究全沙不足以准确掌握坝下游不平衡输沙规律. 故此处 首先给出了荆江河段概况, 然后根据不同粒径悬沙的输移特性, 以 $0.125 \mathrm{~mm}$ 为分界粒径将其分为细沙 ( $d<$ $0.125 \mathrm{~mm})$ 和粗沙 $(d>0.125 \mathrm{~mm})$ 两组, 研究荆江段全沙及分组悬移质输沙量随时间及沿程的变化特点.

\section{1 河段概况}

长江中游荆江段进口位于三峡大坝下游约 $102 \mathrm{~km}$ 处, 上起枝城, 下迄城陵矶, 河段长 $347.2 \mathrm{~km}$. 根据地 理环境与河道特性不同, 以藕池口为界将其分为上、下两段, 其中上荆江长度为 $171.7 \mathrm{~km}$, 为微弯分汉型河 
道, 下荆江长 $175.5 \mathrm{~km}$, 为典型的蜿蜒型河道 (图 1). 荆江干流设有枝城、沙市和监利 3 个水文站, 南岸有松 滋、太平和藕池三口分流汇人洞庭湖. 江口以上为卵石夹沙河床, 河床中有卵砾石洲滩分布, 江口以下为沙 质河床, 床沙中值粒径约为 $0.18 \mathrm{~mm}$, 卵石层深埋至床面以下 ${ }^{[15]}$. 三峡工程运用后 (2003-2017 年), 荆江段 进口枝城站多年平均水量约为 4133 亿 $\mathrm{m}^{3} / \mathrm{a}$, 较蓄水前 (1994- 2002 年) 的 4421 亿 $\mathrm{m}^{3} / \mathrm{a}$ 减少了 $7 \%$; 而年均 沙量 ( 0.43 亿 $\mathrm{t} / \mathrm{a}$ ) 较蓄水前 ( 3.48 亿 $\mathrm{t} / \mathrm{a}$ ) 骤减 $88 \%$, 河段处于严重的次饱和输沙状态, 河床发生了显著冲刷. 此外, 荆江段汛期 (5-10 月) 集中输沙现象十分明显 (占全年来沙量的 86\% 99\%), 故认为该河段泥沙输移 及河床调整主要发生在汛期,非汛期输沙的影响暂不考虑.

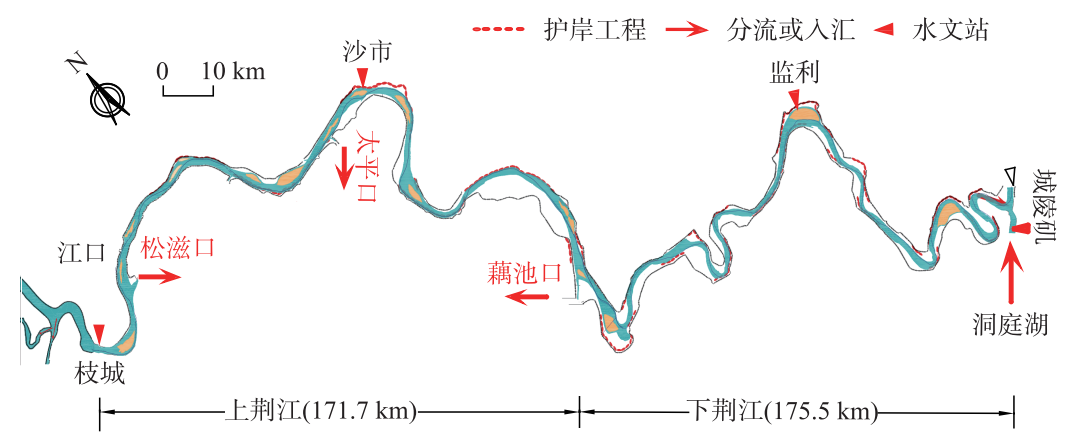

图 1 荆江河段示意图

Fig.1 Sketch of the Jingjiang Reach

\section{2 悬移质输沙量变化}

1.2.1 悬移质输沙量随时间的变化 根据三峡水库运用情况, 将荆江河段水沙变化分为 4 个阶段: 三峡水库 运用前 (1994-2002 年), 荆江河段总体接近冲淤平衡状态 ${ }^{[16]} ; 2003-2008$ 年三峡水库处于围堰发电以及初 期运行状态, 水流处于次饱和状态; 2008 年汛后开始 $175 \mathrm{~m}$ 试验性蓄水, 河道来沙量进一步降低; 2013 年开 始,溪洛渡水电站投人运行, 荆江河段来沙量继续减少,河床持续冲刷.

受长江上游干支流水电站的陆续运用、水土保持工程的不断实施、生态环境的持续改善以及三峡水库 运用方式的改变等影响, 荆江河段悬移质输沙量持续降低 ${ }^{[17-19]}$. 图 2 给出了荆江干流 3 个水文站 (枝城、沙 市和监利) 全沙及分组沙在三峡工程运用前后的多年平均汛期输沙量. 三峡水库运用后, 汛期进人该河段 (枝城站) 的各粒径组沙量均持续减小, 其中细沙较蓄水前降幅较大, 而粗沙降幅较小. 三峡水库运用初期 (2003-2008 年) 枝城站全沙和细沙多年平均输沙量分别为 0.73 亿和 0.59 亿 $\mathrm{t} / \mathrm{a}$, 较蓄水前分别下降了 $80 \%$ 和 $83 \%$, 而粗沙输沙量 ( 0.14 亿 $\mathrm{t} / \mathrm{a}$ ) 较蓄水前 $(0.22$ 亿 $\mathrm{t} / \mathrm{a}$ ) 仅下降了 $35 \%$. 这主要是因为从宜昌到枝城段床 沙较粗, 在次饱和水流冲刷过程中, 河床中较多的粗沙补给到水流中, 致使枝城站粗沙恢复程度较高.

汛期从该河段输出 (监利站) 的细沙量同样持续下降, 而输出的粗沙量随时间先增后减. 在上述 4 个阶 段, 监利站多年平均的细沙输沙量分别为 2.70 亿、0.60 亿、0.39 亿和 0.13 亿 $\mathrm{t} / \mathrm{a}$. 三峡水库运用初期 ( $2003-$ 2008 年), 监利站多年平均的汛期粗沙输沙量为 0.27 亿 $\mathrm{t} / \mathrm{a}$, 较蓄水前的 0.20 亿 $\mathrm{t} / \mathrm{a}$ 增大了 $35 \%$; 之后 $175 \mathrm{~m}$ 试验蓄水期 (2009-2013 年) 和溪洛渡水电站运行后的时期 (2014- 2017 年) 监利站相应的平均粗沙输沙量 均为 0.15 亿 $\mathrm{t} / \mathrm{a}$, 较蓄水初期略有减小.

1.2.2 悬移质输沙量沿程变化 次饱和水流持续冲刷河床, 荆江河段各粒径组沙量沿程都有不同程度的恢 复, 尤其粗沙部分 (图 2a c ). 例如, 2014-2017 年间, 枝城、沙市和监利站多年平均的细沙输沙量分别为 0.07 亿、 0.11 亿和 0.13 亿 $\mathrm{t} / \mathrm{a}$, 粗沙分别为 0.01 亿、 0.06 亿和 0.15 亿 $\mathrm{t} / \mathrm{a}$, 均沿程明显增大. 究其原因, 主要是 由于床沙的补给. 考虑三口分流的作用, 采用输沙量法计算了不同阶段枝城一监利河段的冲淤量, 即河床的 补给量 (图 2d). 可知, 2003-2017 年荆江枝城一监利段多年平均细沙和粗沙冲刷量分别为 0.12 亿和 0.14 亿 $\mathrm{t} / \mathrm{a}$, 粗沙冲刷量相对较大. 这也可以解释前文所述荆江段出口(监利站)不同粒径悬沙量随时间变化存在差 异的原因. 细沙在该河段恢复的沙量小于上游来沙随时间减少的沙量, 故在监利站细沙量仍随时间呈明显 的下降趋势; 然而, 蓄水初期 (2003-2008 年)粗沙在该河段内的恢复量超过了上游来沙的减小量, 故该时段 

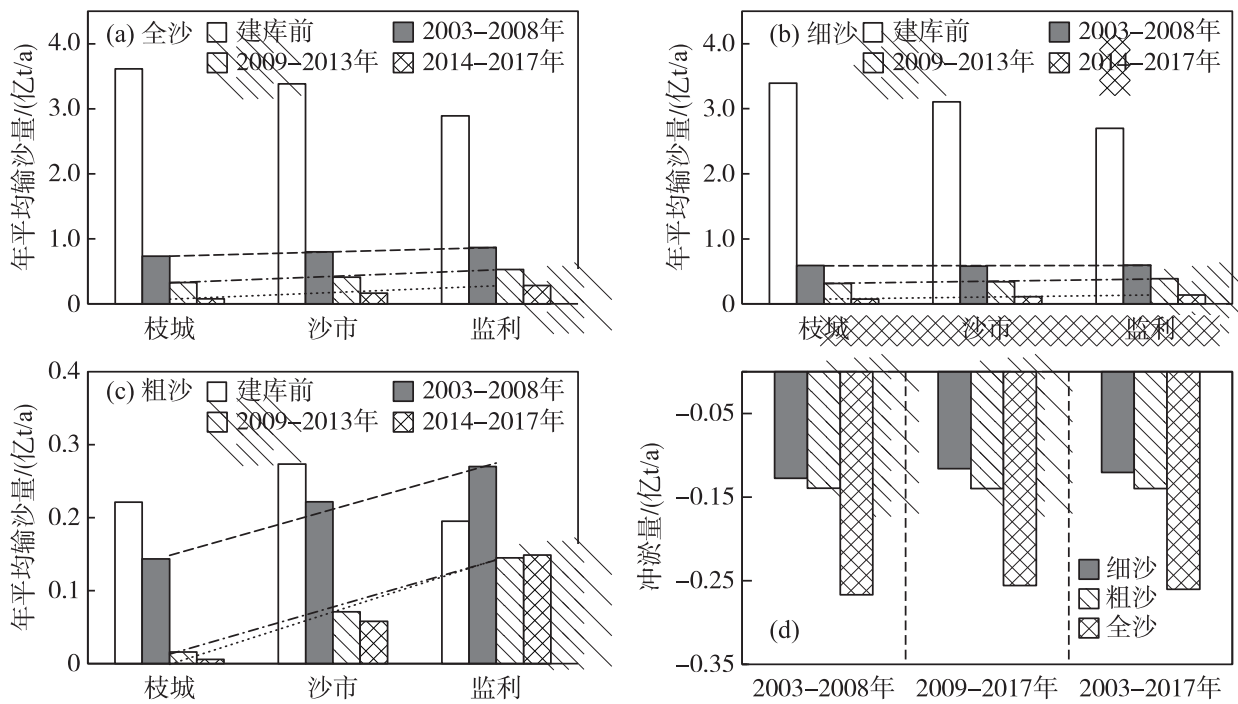

图 2 荆江段不同时期各水文站输沙量及荆江段分组沙冲淤量: (a) 全沙; (b) 细沙; (c) 粗沙; (d) 冲淤量

Fig.2 Average annual suspended sediment discharges for different fractions at hydrometric stations in the Jingjiang Reach and the channel scour volume in the reach

监利站多年平均的粗沙输沙量超过了蓄水前的水平.

进一步分析可知, 不同粒径组泥沙恢复程度的差异主要与荆江段的床沙组成有关. 图 3 给出了 3 个水 文站 (枝城、沙市和监利) 不同年份的汛后床沙级配. 该河段床沙中细沙所占的比例很小, 2003 年 3 个水文 站床沙中值粒径小于 $0.125 \mathrm{~mm}$ (细沙) 占比分别仅为 $1.1 \% 、 4.3 \%$ 和 $37.2 \%$. 且随着冲刷历时的增大, 细沙占 比仍在不断减小,到 2017 年细沙颗粒占比分别降至 $0.2 \% 、 0.2 \%$ 和 $4.0 \%$, 因此河床不能为水流提供充足的细 沙补给, 故其恢复程度较小. 然而该河段河床中粗沙的含量较大, 且该比例呈逐年增大的趋势, 河床中有足 够的粗沙提供补给, 因此粗沙恢复程度较大.
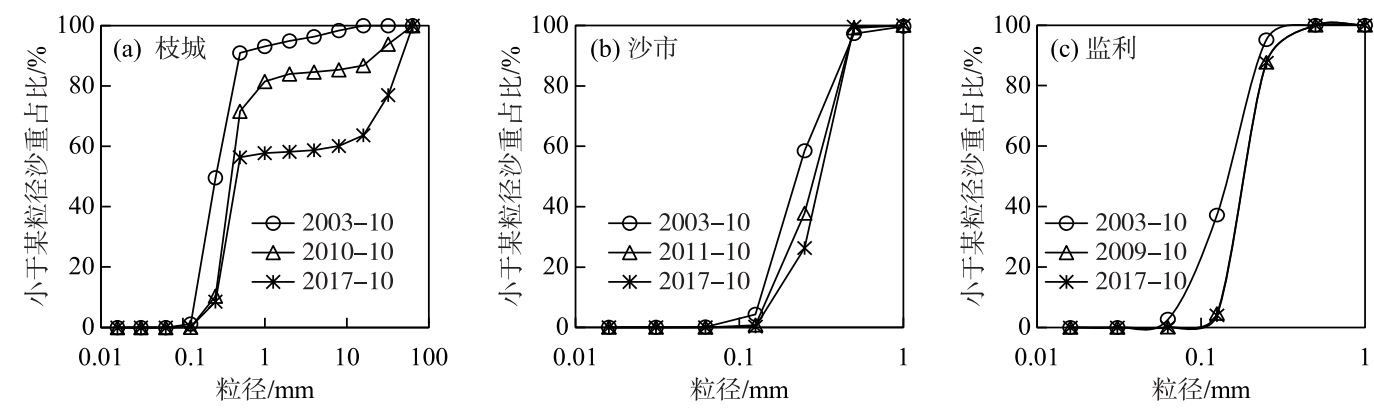

图 3 荆江段各水文站不同年份实测床沙级配

Fig.3 Measured bed-material grain-size distributions at hydrometric stations in the Jingjiang Reach

\section{3 悬移质组成变化}

悬沙组成是影响河床冲淤的重要因素. 在不平衡输沙过程中,悬沙与床沙发生交换, 由于相同水流条件 下不同粒径泥沙的冲刷效率不同, 导致悬沙级配也会发生变化 ${ }^{[20]}$. 为充分了解坝下游非均匀沙不平衡输移 规律,有必要研究悬移质组成的变化情况.

为直观表示荆江段悬移质组成的变化情况, 图 4 给出了枝城、沙市和监利站汛期平均的悬移质中值粒 径逐年变化过程. 可知, 三峡水库运用前 (1994-2002 年), 荆江段各水文站悬移质中值粒径相差不大, 且随 
时间无明显变化,其数值均在 $0.010 \mathrm{~mm}$ 左右. 蓄水后 (2003-2017 年), 该河段各水文站悬移质均有不同程 度的粗化, 对比 3 条趋势线切线的斜率可知, 悬移质粗化程度为监利 $>$ 沙市 $>$ 枝城. 枝城站悬移质中值粒径由 2003 年的 $0.011 \mathrm{~mm}$ 增大到了 2017 年的 $0.016 \mathrm{~mm}$, 沙市站由 2003 年的 $0.017 \mathrm{~mm}$ 增大到了 2017 年的 0.053 $\mathrm{mm}$, 而监利站由 2003 年的 $0.017 \mathrm{~mm}$ 增大到了 $0.176 \mathrm{~mm}$, 增幅最大, 粗化最为显著. 此外, 该河段悬移质中 值粒径沿程增大, $2003-2017$ 年 3 个水文站多年平均悬移质中值粒径分别为 $0.01 、 0.02$ 和 $0.07 \mathrm{~mm}$. 这主要 是由于次饱和水流的冲刷作用,使荆江段较粗的床沙颗粒补给到挟沙水流中.



图 4 荆江段各水文站悬移质中值粒径的逐年变化过程

Fig.4 Temporal variations in medium diameters of suspended sediment at each hydrometric station of the Jingjiang Reach

\section{2 分组悬沙恢复效率计算及结果分析}

上节已从冲淤量的角度分析了荆江段不平衡输沙过程中分组沙的恢复情况, 但由于水流中不同粒径泥 沙的占比不同, 不能简单根据冲淤量判断悬沙恢复的程度. 韩其为等 ${ }^{[21]}$ 从含沙量与挟沙力的关系出发, 认 为含沙量向挟沙力靠拢的过程为含沙量的恢复过程. 在同一水流条件下, 细沙的挟沙力通常远大于粗沙, 在 细沙和粗沙的河床冲刷补给量相近时, 可能粗沙已基本达到平衡状态, 而细沙仍处于严重非饱和状态, 单纯 分析不同粒径组泥沙的河床冲淤量不能很好地反映非均匀沙的恢复效率. 因此,此处采用研究河段的河床 冲淤量与进人该河段的沙量之比来表征非均匀沙的恢复效率. 需说明的是, 通常情况下由于泥沙及地形测 量误差、断面布置以及河道采砂的影响, 地形法和输沙量法计算所得冲淤成果存在一定的差异. 但是由于目 前采用地形法难以准确确定分组沙的冲淤量, 而本文研究重点为不同粒径组悬沙的恢复特性, 故采用了输 沙量法确定分组沙冲淤量. 在河槽形态调整不大且床沙级配随时间变化不大的情况下, 进口水沙条件是影 响悬移质泥沙输移及恢复的主要因素. 故此处建立荆江段非均匀悬沙恢复效率与河段来水来沙条件之间的 经验关系,用以分析来水来沙条件对恢复效率的影响特点.

\section{1 计算方法}

2.1.1 恢复效率的概念及计算 将荆江段内第 $k$ 粒径组沙的河床冲淤量 (淤积为正, 冲刷为负) 与进人该河 段第 $k$ 粒径组悬沙量的比值记作第 $k$ 粒径组悬沙的恢复效率 $\left(\lambda_{k}\right)$. 考虑到荆江三口 (松滋、太平、藕池口) 分流分沙作用, 以枝城站汛期第 $k$ 粒径组悬沙的来沙量 $W_{s k, \mathrm{ZC}}$ 作为进人荆江段的第 $k$ 粒径组沙量 $W_{s k, \mathrm{in}}$, 以监 利汛期第 $k$ 粒径组悬沙的沙量 $W_{s k, \mathrm{JL}}$ 与三口汛期第 $k$ 粒径组悬沙的分沙量 $W_{s k \text {, т }}$ 之和作为荆江河段第 $k$ 粒径 组悬沙的输沙量 $W_{s k, \text { out }}$, 由此可得荆江段第 $k$ 粒径组悬沙的恢复效率, 相应计算公式如下:

$$
\lambda_{k}=\left(W_{s k, \text { in }}-W_{s k, \text { out }}\right) / W_{s k, \text { in }}=\left(W_{s k, \mathrm{ZC}}-\left(W_{s k, \mathrm{JL}}+W_{s k, \mathrm{TH}}\right)\right) / W_{s k, \mathrm{ZC}}
$$

显然, $\lambda_{k}>0$ 时, 第 $k$ 粒径组悬沙处于超饱和状态, 河床淤积; $\lambda_{k}<0$ 时, 第 $k$ 粒径组悬沙处于次饱和状 态, 河床冲刷; $\lambda_{k}$ 接近 0 时悬移质近似处于平衡输沙状态, 河床不冲不淤. 在不平衡输沙状态下, $\lambda_{k}$ 的绝对 值越大, 悬沙向平衡状态恢复的程度越高.

2.1 .2 来水来沙条件计算 来水来沙条件是影响河床演变的主要因素之一 ${ }^{[22]}$. 对于冲积河流,通常采用来沙 系数来表征来水来沙条件 ${ }^{[23]}$. 来沙系数一般指特定时段内平均含沙量与相应流量的比值 (为避免其数值过 小, 按 $10^{4}$ 放大), 其代表了单位流量含沙量的大小. 分组沙来沙系数的计算公式为:

$$
\xi_{k}=\left(\bar{S}_{k} / \bar{Q}\right) \times 10^{4}
$$

式中, $\xi_{k}$ 为第 $k$ 粒径组悬沙的来沙系数; $\bar{Q}$ 为枝城站汛期平均流量 $\left(\mathrm{m}^{3} / \mathrm{s}\right), \bar{S}_{k}$ 为相应第 $k$ 粒径组悬沙的含 
沙量 $\left(\mathrm{kg} / \mathrm{m}^{3}\right)$. 来沙系数 $\xi_{k}$ 越大, 河道越容易淤积, 反之越有利于冲刷 ${ }^{[24]}$. 本研究采用枝城站汛期实测数据 计算来沙系数,代表荆江段的来水来沙条件.

\section{2 计算结果}

2.2.1 非均匀沙恢复效率 根据 1994-2017 年实测水沙数据, 采用式 (1) 计算所得荆江段细沙及粗沙恢复 效率, 如图 5a 所示. 可知: 三峡水库运用前, 荆江段细沙及粗沙恢复效率差别不大, 且其绝对值都接近 0 ; 三 峡水库运用后, 研究河段细沙及粗沙恢复效率均变为负值, 且其绝对值均一定程度增大, 其中粗沙恢复效率 的绝对值远大于细沙, 且随时间呈增大趋势. 这主要是由于三峡水库运用前, 荆江段总体接近输沙平衡状 态, 悬移质输沙量沿程变化不大, 故多年平均汛期细沙和粗沙恢复效率绝对值均很小, 其数值均为 0.04 . 三 峡水库运用后, 荆江段各粒径组悬沙的恢复效率绝对值较蓄水前均明显增大, 这主要是由于水库运用后, 各 粒径组悬沙均处于严重次饱和输沙状态, 为了恢复输沙平衡状态, 河床显著冲刷, 各粒径组悬移质含沙量沿 程恢复. 然而, 不同粒径组悬沙恢复效率存在显著差异. 汛期粗沙恢复效率介于 $-53.43 \sim 0.37$ 之间, 其多年平 均值为 -14.07 , 且其绝对值随冲刷历时增加呈明显的增大趋势; 而细沙恢复效率绝对值远小于粗沙, 变化范 围为 $-1.56 \sim-0.17$, 其绝对值也随冲刷历时增加但增幅不大. 这主要是因为床沙中细沙占比很小, 在次饱和 冲刷过程中, 河床不能为水流提供充足的细沙补给, 因此细沙的恢复存在一定限制, 而床沙中粗沙占比较 大, 恢复不存在限制, 故其恢复效率绝对值增幅也更大.

2.2.2 来沙系数 三峡水库运用后 (2003-2017 年), 由于水库的拦蓄作用, 三峡大坝下泄水流的含沙量大幅 降低, 而下泄流量变幅不大, 因此荆江段来沙系数急剧减小 (图 5b). 蓄水后, 多年平均汛期全沙来沙系数仅 为 $0.07(\mathrm{~kg} \cdot \mathrm{s}) / \mathrm{m}^{6}$, 较蓄水前的 $0.50(\mathrm{~kg} \cdot \mathrm{s}) / \mathrm{m}^{6}$ 减小了 $86 \%$; 细沙来沙系数为 $0.06(\mathrm{~kg} \cdot \mathrm{s}) / \mathrm{m}^{6}$, 较蓄水前 $\left(0.47(\mathrm{~kg} \cdot \mathrm{s}) / \mathrm{m}^{6}\right)$ 减小了 $87 \%$; 粗沙为 $0.01(\mathrm{~kg} \cdot \mathrm{s}) / \mathrm{m}^{6}$, 相比蓄水前 $\left(0.03(\mathrm{~kg} \cdot \mathrm{s}) / \mathrm{m}^{6}\right)$ 下降了 $66 \%$. 整体上, 各粒径组悬沙来沙系数均大幅下降,水流处于严重的次饱和状态, 河床发生剧烈冲刷.
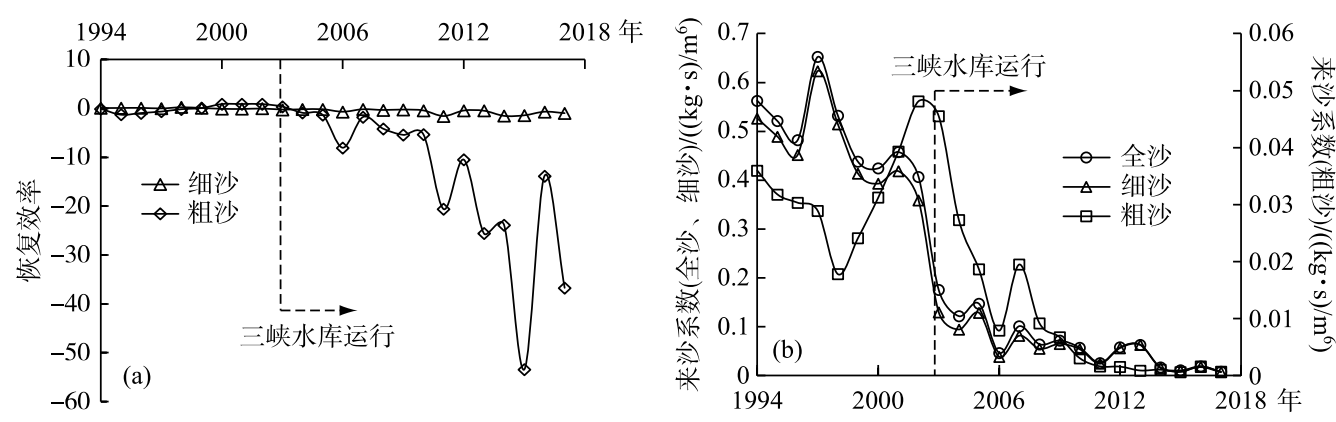

图 5 1994-2017 年分组悬沙的恢复效率与来沙系数:恢复效率 (a) ; 来沙系数 (b)

Fig.5 Calculated results for grouped sediment from 1994 to 2017: recovery efficiency $(\mathrm{a})$; incoming sediment coefficient (b)

\section{3 非均匀沙恢复效率与来水来沙条件的关系}

当河段来水来沙条件发生波动时, 河床会做出自动调整来适应这种变化. 在坝下游河段的不平衡输沙 过程中, 床沙级配随时间变化不大的情况下, 来水来沙条件是最直接影响到非均匀沙沿程恢复情况 (也即分 组沙的恢复效率) 的因素, 因此有必要研究两者之间的关系, 从而提高对于坝下游含沙量恢复特性的认识. 根据上述计算所得 2003-2017 年恢复效率和来沙系数结果,建立了全沙及分组沙恢复效率与来沙系数的相 关关系. 采用对数关系拟合, 两者决定系数最高, 结果如图 6 所示.

全沙、细沙和粗沙恢复效率与来沙系数的决定系数分别为 $0.89 、 0.67$ 和 0.69 , 总体上荆江段分组沙恢复 效率可较好地对来水来沙条件作出响应. 其中细沙及粗沙的决定系数较全沙有所减小, 这主要是由于悬沙 级配测量存在一定误差. 在来沙系数很小也即水流不饱和程度很高时, 恢复效率为负值且其绝对值很大, 河 床处于冲刷状态; 当来沙系数增大到一定程度时, 也即水流由次饱和状态变为超饱和状态时, 恢复效率由负 变为正值, 悬移质淤积到河床上, 这说明该计算关系与实际物理过程相符, 具有合理性. 此外, 根据拟合所得 
关系式可求得一个恢复效率为 0 (冲淤平衡) 时的来沙系数临界值, 全沙、细沙和粗沙的临界值分别为 0.142 、 0.161 和 $0.019(\mathrm{~kg} \cdot \mathrm{s}) / \mathrm{m}^{6}$. 可知, 粗沙由淤积状态变为冲刷状态的临界来沙系数明显小于细沙, 这主要是由 于泥沙粒径越大, 越难将其从河床上冲起, 相应水流需达到的次饱和程度也越高 (也即来沙系数越小).
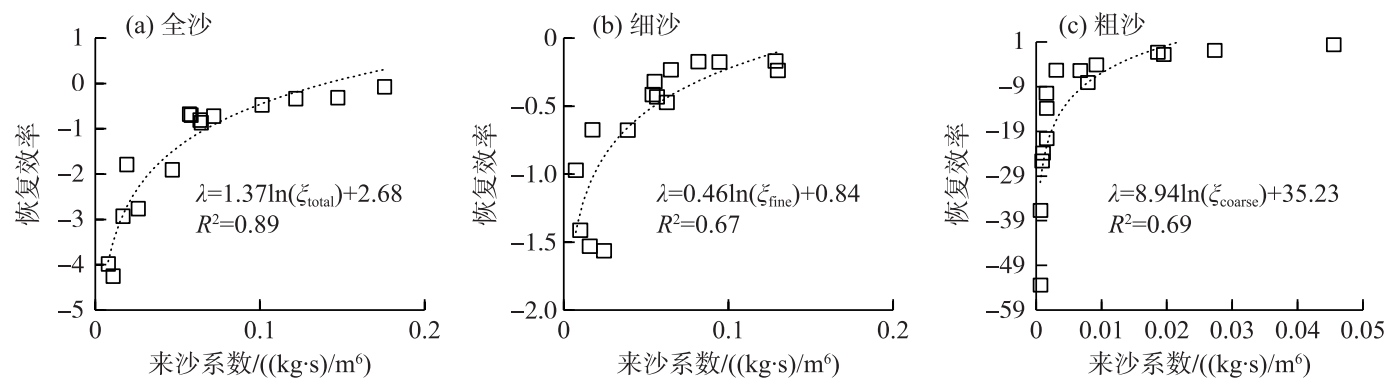

图 6 非均匀沙恢复效率与来沙系数的关系

Fig.6 Relationship between the recovery efficiency for each group and the incoming sediment coefficient

\section{3 结论}

根据 1994-2017 年荆江段实测水沙资料, 分析了三峡水库运用前后荆江段非均匀悬沙恢复特点; 提出 了恢复效率的概念用来表征悬移质沿程恢复的程度, 并计算了荆江段不同粒径组悬沙的恢复效率; 建立了 非均匀沙恢复效率与来沙系数的关系, 定量确定了来水来沙条件对荆江段悬沙沿程恢复的影响. 主要结论 如下:

1) 1994-2017 年进人荆江段的各粒径组沙量均持续减小, “清水” 持续冲刷河床, 使得各粒径组悬沙量 沿程恢复. 由于荆江段床沙中粗沙 $(d>0.125 \mathrm{~mm})$ 含量大, 而细沙 $(d<0.125 \mathrm{~mm})$ 含量小, 使得细沙恢复程度 较低而粗沙恢复程度较高, 导致悬沙随时间及沿程均显著粗化.

2) 三峡水库运用前, 荆江段恢复效率绝对值接近 0 , 表明河段冲淤变幅不大; 蓄水后, 各粒径悬移质恢复 效率绝对值均明显增大,其中粗沙恢复效率绝对值远大于细沙, 且随冲刷历时增加呈明显增大趋势. 不同粒 径悬移质泥沙恢复效率均与来沙系数呈正相关关系, 且决定系数较高 $\left(R^{2}=0.67 \sim 0.89\right)$, 因此在研究时段内 非均匀悬沙的恢复效率主要受到进口来水来沙条件的影响.

\section{4 参考文献}

[ 1 ] Termini D. Non-uniform sediment transport estimation in non-equilibrium situations: Case studies. Procedia Engineering, 2014, 70: 1639-1648. DOI: 10.1016/j.proeng.2014.02.181.

[ 2 ] Liu JM, Wang GQ, Wang SQ. Study on non-equilibrium sediment transport in the sandy river erosion. Advances in Water Science, 2003, 14(5): 563-568. [刘金梅, 王光谦, 王士强. 沙质河道冲刷不平衡输沙机理及规律研究. 水科学进 展, $2003,14(5): 563-568$. ]

[ 3 ] Xu ZM, Xu XY, Li AQ et al. Flood control effect of the fluvial process in the middle and lower reaches of the Yangtze River. Advances in Water Science, 2020, 31(3) : 366-376. [徐照明, 徐兴亚, 李安强等. 长江中下游河道冲淤演变的防 洪效应. 水科学进展, 2020, 31(3): 366-376.]

[ 4 ] Liu C, Sui JY, He Y et al. Changes in runoff and sediment load from major Chinese rivers to the Pacific Ocean over the period 1955-2010. International Journal of Sediment Research, 2013, 28(4) : 486-495. DOI: 10.1016/S1001-6279( 14) 60007-X.

[ 5 ] Zhang QS. Diffusion process of sediment in open channel and its application. Journal of Sediment Research, 1980, (1): 37-52. [张启舜. 明渠水流泥沙扩散过程的研究及其应用. 泥沙研究, 1980, (1) : 37-52.]

[ 6 ] Han QW, He MM. 2 D nonequilibrium transportation equation of nonuniform suspended load and its boundary condition. Journal of Hydraulic Engineering, 1997, 28(1) : 2-11. [韩其为, 何明民. 论非均匀悬移质二维不平衡输沙方程及其 边界条件. 水利学报, $1997,28(1): 2-11$.] 
[ 7 ] Qian N, Zhang R eds. Bed evolution. Beijing: Science Press, 1987. [钱宁, 张仁. 河床演变学. 北京: 科学出版 社, 1987.]

[ 8 ] Han QW ed. Reservoir sedimentation. Beijing: Science Press, 2003. [韩其为. 水库淤积. 北京: 科学出版社, 2003.]

[ 9 ] Liu JM, Wang SQ, Wang GQ. A study on non-equilibrium sediment transport process of long distanceerosion in fluvial river. Journal of Hydraulic Engineering, 2002, 33(2): 47-53. [刘金梅, 王士强, 王光谦. 冲积河流长距离冲刷不平衡 输沙过程初步研究. 水利学报, $2002, \mathbf{3 3}(2): 47-53$.

[10] Li YT, Chen F. Non-uniform sediment transport downstream from reservoir. Transactions of Tianjin University, 2008,14 (4) : 263-270. DOI: 10.1007/s12209-008-0046-2.

[11] Chen F, Li YT, Tang JW et al. Analysis of group-sized sediment transport downstream a reservoir. Journal of Hydroelectric Engineering, 2010, 29(1) : 164-170. [陈飞, 李义天, 唐金武等. 水库下游分组沙冲淤特性分析. 水力发电学报, $2010,29(1): 164-170$.]

[12] Guo XH, Li YT, Qu G et al. Analysis of sediment transport in Middle Yangtze River after filling of the Three Gorges Reservoir. Journal of Sediment Research, 2014, (5): 11-17. [ 郭小虎, 李义天, 渠庚等. 三峡工程蓄水后长江中游泥沙 输移规律分析. 泥沙研究, 2014, (5): 11-17.]

[13] Yang YP, Zhang MJ, Li SZ et al. Transport patterns of the coarse and fine sediments and its causes in the downstream of the Three Gorges Dam. J Lake Sci, 2017, 29(4) : 942-954. DOI: 10.18307/2017.0418. [杨云平, 张明进, 李松喆等. 三峡大坝下游粗细颗粒泥沙输移规律及成因. 湖泊科学, 2017, 29(4) : 942-954.]

[14] Chen JG, Zhou WH, Yuan YP. Transportation and adjustment of different grain sized sediment along the lower Yellow River under typical operation modes of Sanmenxia reservoir. Journal of Sediment Research, 2002, (2) : 15-22. [陈建国, 周 文浩, 袁玉萍. 三门峡水库典型运用时段黄河下游粗细泥沙的输移和调整规律. 泥沙研究, 2002, (2): 15-22.]

[15] Xia JQ, Lin FF, Zhou MR et al. Bank retreat processes and characteristics in the Jingjiang Reach after the Three Gorges Project operation. Advances in Water Science, 2017, 28(4) : 543-552. [夏军强, 林芬芬, 周美蓉等. 三峡工程运用后 荆江段崩岸过程及特点. 水科学进展, 2017, 28(4): 543-552.]

[16] Xu QX. Study of sediment deposition and erosion patterns in the middle and downstream Changjiang mainstream after impoundment of TGR. Journal of Hydroelectric Engineering, 2013, 32(2) : 146-154. [许全喜. 三峡工程蓄水运用前后长 江中下游干流河道冲淤规律研究. 水力发电学报, 2013, 32(2) : 146-154.]

[17] Chen J. An approach on flood control strategy in middle and lower reaches of Yangtze River after the completion of the Three Gorges Dam project. Advances in Water Science, 2014, 25(5): 745-751. [陈进. 三峡水库建成后长江中下游防 洪战略思考. 水科学进展, 2014, 25(5): 745-751.]

[18] Lyu YW, Zheng S, Tan GM et al. Effects of Three Gorges Dam operation on spatial distribution and evolution of channel thalweg in the Yichang-Chenglingji Reach of the Middle Yangtze River, China. Journal of Hydrology, 2018, 565: 429442. DOI: 10.1016/j.jhydrol.2018.08.042.

[19] Wang YK, Rhoads BL, Wang D et al. Impacts of large dams on the complexity of suspended sediment dynamics in the Yangtze River. Journal of Hydrology, 2018, 558: 184-195. DOI: 10.1016/j.jhydrol.2018.01.027.

[20] Han QW. A study of the non-equilibrium transportation of non-uniform suspended load. Chinese Science Bulletin, 1979,24 (17)：804-808. [ 韩其为. 非均匀悬移质不平衡输沙的研究. 科学通报, 1979, 24(17)：804-808.]

[21] Han QW. Only deposition in river reach from Chenglingji to Wuhan after impounding of TGR? —Response on "Discussion on the flood control capacity of the middle Yangtze River after the impoundment of Three Gorges Reservoir”. Journal of Hydroelectric Engineering, 2006, 25(6) : 79-90. [ 韩其为. 三峡水库运行后城汉河段会只淤不冲吗? 一一对 “关于三 峡工程对城陵矶防洪能力影响有关研究的讨论”的讨论. 水力发电学报, 2006, 25(6) : 79-90.]

[22] Phillips JD. Toledo Bend reservoir and geomorphic response in the lower Sabine River. River Research and Applications, 2003, 19(2) : 137-159. DOI: 10.1002/rra.702.

[23] Shen GQ, Jiang NQ, Li Y et al. Research on water volume of sediment transport and the calculating method in the lower Yellow River. Advances in Water Science, 2006, 17(3) : 407-413. [申冠卿, 姜乃迁, 李勇等. 黄河下游河道输沙水量 及计算方法研究. 水科学进展, 2006, 17(3): 407-413.]

[24] Wu BS, Zhang YF, Xia JQ. Variation of bank-full area at Gaocun station in the Lower Yellow River. Journal of Sediment Research, 2008, (2) : 34-40. [吴保生, 张原锋, 夏军强. 黄河下游高村站平滩面积变化分析. 泥沙研究, 2008, (2) : 34-40.] 